\title{
Near band edge polarization dependence as a probe of structural symmetry in GaAs/AIGaAs quantum dot structures
}

\author{
T. Tanaka, J. Singh, ${ }^{a)}$ and Y. Arakawa \\ Research Center for Advanced Science and Technology, University of Tokyo, 4-6-1 Meguro-ku, \\ Tokyo 153, Japan \\ P. Bhattacharya \\ Department of Electrical Engineering and Computer Science, The University of Michigan, \\ Ann Arbor, Michigan 48109
}

(Received 9 July 1992; accepted for publication 19 November 1992)

In order to obtain the polarization dependence of optical transitions in GaAs/AlGaAs quantum dot structures, a three-dimensional Schrödinger equation describing the heavy hole-light hole mixing via a $\mathbf{k} \cdot \mathbf{p}$ formalism is solved. The polarization dependence is investigated as a function of the symmetry of the quantum dot, indicating that the polarization dependence is very sensitive to the symmetry of the confining structure. We also examine how the optical properties of the quantum dot evolve towards those of a quantum wire by allowing one axis of the dot to increase.

Recently there has been an increasing interest in subtwo-dimensional structures such as quantum dots and quantum wires. ${ }^{1-4}$ The motivation for the interest is due to the greater degree of tailoring of the electronic spectra that is possible in such structures. In particular the electronic density of states can be greatly altered in such structures. The altered density of states can be exploited for both electronic and optoelectronic devices. Additionally, the nature of the valence band states can be dramatically altered to provide tailorable polarization dependent effects. Quantum dot structures can be fabricated in semiconductor heterostructure technology by a combination of lithography, etching, and epitaxial techniques. They can also be produced in certain glass manufacturing processes where quantum dots as small as $\sim 50 \AA$ diam can be imbedded in the glass. It has also been shown that in quantum wires which have interfacial disorder, the electron and hole states are localized, and for low lying carrier states, the quantum wire appears to be a collection of quantum dots. ${ }^{4}$ It is important to develop techniques which can identify the symmetry of the quantum dot structures and also allow design of quantum dots with tailorable polarization selectivity.

In quantum well and quantum wire structures, near band edge polarization dependence is known to have a strong influence of confinement and dimensionality. ${ }^{5-8}$ This polarization dependence is useful for designing special devices. In quantum dot structures, there is little understanding of the optical properties, particularly the polarization dependence. Such studies are important for both future optical devices as well as for the purpose of optically characterizing quantum dots. To address this problem one needs to solve a three-dimensional Schrödinger equation which includes the heavy hole (HH)-light hole (LH) interactions in the valence band description. In this letter we

\footnotetext{
a) Permanent address: Department of Electric Engineering and Computer Science, The University of Michigan, Ann Arbor, MI 48109.
}

report results based on such a solution. Our results show a strong sensitivity of polarization dependence on the symmetry of the quantum dot.

The conduction band states in the GaAs/AlGaAs system are well described by an isotropic effective mass. The states can thus be described by a single band Schrödinger equation. The valence band states, however, are not so simple due to the very strong coupling between the HH and LH states. This coupling leads to strongly nonparabolic and anisotropic bands and in the case of quantum well and quantum wire structures leads to bands with negative hole mass. To solve for the valence band spectra in quantum dots one therefore needs to solve a Schrödinger equation which is capable of including the HH-LH coupling in the three-dimensional confined structures. The Kohn-Luttinger formalism ${ }^{9}$ has been widely used to describe valence band states. This approach describes the hole masses and density of states quite accurately and can be conveniently applied to the three-dimensional confined structures where periodic boundary condition is not available.

In the three-dimensional confined heterostructures, the Kohn-Luttinger formalism yields the 4-band Schrödinger equation (describing the HH and $\mathrm{LH}$ bands),

$$
\left[\begin{array}{cclc}
H_{\mathrm{hh}} & c & b & 0 \\
c^{*} & H_{\mathrm{lh}} & 0 & -b \\
b^{*} & 0 & H_{\mathrm{lh}} & c \\
0 & -b^{*} & c^{*} & H_{\mathrm{hh}}
\end{array}\right]\left[\begin{array}{l}
g_{n}^{1}(x, y, z) \\
g_{n}^{2}(x, y, z) \\
g_{n}^{3}(x, y, z) \\
g_{n}^{4}(x, y, z)
\end{array}\right]=E_{n}\left[\begin{array}{c}
g_{n}^{1}(x, y, z) \\
g_{n}^{2}(x, y, z) \\
g_{n}^{3}(x, y, z) \\
g_{n}^{4}(x, y, z)
\end{array}\right]
$$

where $g_{n}^{v}(x, y, z)$ are the envelope functions of the $n$th eigenstates and the basis $u_{v}(v=1,2,3,4)$ are those of the angular momentum $\mathbf{J}, \mathbf{J}_{z}:|3 / 2,3 / 2\rangle,|3 / 2,-1 / 2\rangle,|3 / 2,1 / 2\rangle$, and $|3 / 2,-3 / 2\rangle$, respectively. Here, $|3 / 2, \pm 3 / 2\rangle$ and $\mid 3 / 2$, $\pm 1 / 2\rangle$ are the $\mathrm{HH}$ and $\mathrm{LH}$ basis, respectively. The elements in the Hamiltonian are given by 
$H_{\mathrm{hh}}=\frac{-\hbar^{2}}{2 m_{0}}\left[\left(k_{x}^{2}+k_{y}^{2}\right)\left(\gamma_{1}+\gamma_{2}\right)+k_{z}^{2}\left(\gamma_{1}-2 \gamma_{2}\right)\right]+V(x, y, z)$,

$H_{\mathrm{lh}}=\frac{-\hbar^{2}}{2 m_{0}}\left[\left(k_{x}^{2}+k_{y}^{2}\right)\left(\gamma_{1}-\gamma_{2}\right)+k_{z}^{2}\left(\gamma_{1}+2 \gamma_{2}\right)\right]+V(x, y, z)$,

$c=\frac{\sqrt{3} \hbar^{2}}{2 m_{0}}\left[\gamma_{2}\left(k_{x}^{2}-k_{y}^{2}\right)-2 i \gamma_{3} k_{x} k_{y}\right]$

$b=\frac{\sqrt{3} \hbar^{2}}{2 m_{0}}\left(k_{x}-i k_{y}\right) \gamma_{3} k_{z}$

where $\gamma_{1}, \gamma_{2}, \gamma_{3}$ are the Kohn Luttinger parameters and $V(x, y, z)$ is the confining potential arising from band discontinuity in the structure. Since the periodic boundary condition is not available, then $k_{x}, k_{y}, k_{z}$ are replaced by the operator $k_{x}=-i(\partial / \partial x)$, etc. The $4 \times 4$ differential equation is written as a $4 \times N_{x} \times N_{y} \times N_{z}$ matrix equation by discretizing the equation as a difference equation. The three-dimensional space is discretized by a $N_{x}, N_{y}, N_{z}$ mesh. The eigenvalue problem is then solved by standard matrix diagonalizing subroutines. ${ }^{10}$ The convergence of the results are checked by increasing the volume of the barrier region included in the calculation and by reducing the mesh size. In this study, the maximum dimension of the matrix used for calculation is $24024 \times 24024$ and the error of eigenenergy is estimated under $2 \mathrm{meV}$.

For the finite size structure, the eigenspectra is discrete and we calculate the density of states by including a simple Gaussian broadening function. A linewidth of $2 \mathrm{meV}$ is used for our results prescnted here. Once the electron and hole envelope functions $g_{n}^{c}(x, y, z), g_{n}^{v}(x, y, z)$ are known, the polarization dependence is obtained by

$$
\mathbf{P}_{i j}=\sum_{v=1}^{4} \int g_{i}^{c}(x, y, z) g_{j}^{v}(x, y, z) d x d y d z\left\langle u_{c}|\mathbf{p}| u_{\nu}\right\rangle,
$$

where $\left\langle u_{c}|\mathbf{p}| u_{v}\right\rangle$ is the momentum matrix element along the polarization direction between the conduction and valence band basis, and is given by Lawaetz. ${ }^{11}$ Optical transition strength for polarization $\epsilon$ is proportional to the square of $\left|\epsilon \cdot \mathbf{P}_{i j}\right|$.

Here we show results for five structures of GaAs/ $\mathrm{Al}_{0.3} \mathrm{Ga}_{0.7}$ As with dimensions: (a) $70 \AA ̊ \times 70 \AA \times 70 \AA ̊$; (b) $70 \AA \times 70 \AA \times 200 \AA$; (c) $70 \AA \times 70 \AA \times 500 \AA$; (d) 70 $\AA \times \AA 70 \AA \times 1000 \AA$, and (e) $70 \AA \times 100 \AA \times 200 \AA$. The first four structures maintain $x, y$ symmetry and allow us to go from a cubic dot towards a quantum wire. The fifth structure is asymmetric in all axes.

In Fig. 1 we show the conduction band density of states. These results are only shown for comparison since the conduction band problem with isotropic electron mass is rather trivial in the confined structure. In Figs. 1(b)$1(c)$, the discrete peaks are exhibited, which are corresponding to the states $\left(n_{x}, n_{y}, n_{z}\right)=(1,1, \mu)(\mu=1,2,3, \ldots)$, where $n_{x}, n_{y} n_{z}$ are quantum numbers. The density of states is quite well behaved and as the quantum dot tends to-
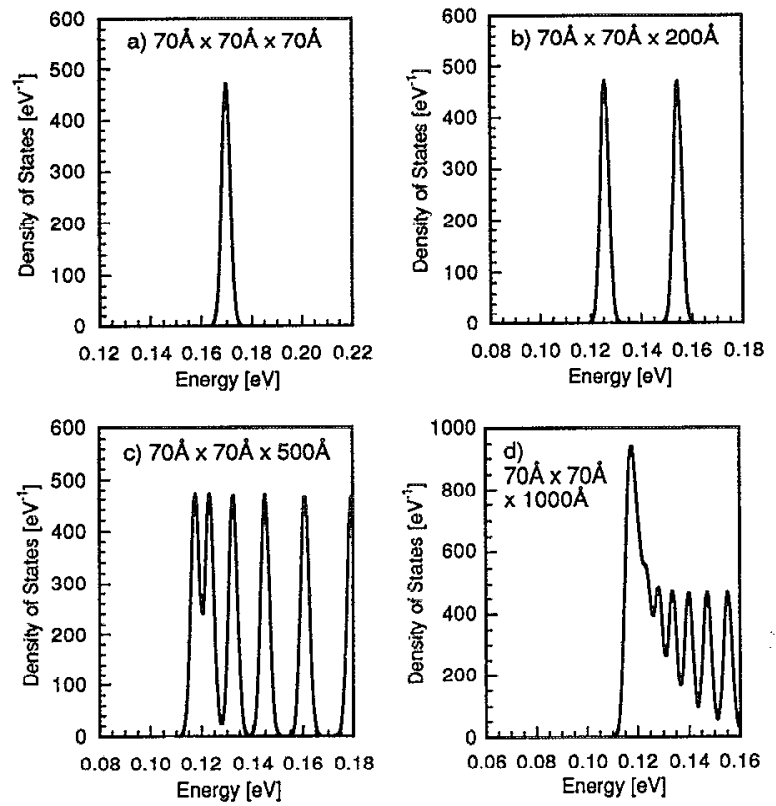

FIG. 1. Density of states for electrons in $\mathrm{GaAs} / \mathrm{Al}_{0.3} \mathrm{Ga}_{0.7} \mathrm{As}$ quantum dots of various sizes. A $2 \mathrm{meV}$ Gaussian broadening function is used to show the density of states.

wards a wire structure, the density of states develops a band edge singularity expected from quantum wire structures.

In Fig. 2 we show the valence band density of states for the same structures. One immediately sees the contrast between the conduction band and valence band density of states. In the valence band since there is very strong $\mathrm{HH}$, LH mixing resulting in highly anisotropic and nonparabolic bands, the spacing of the various energy levels does not follow the relation,

$$
E_{n} \cong \frac{\pi^{2} \hbar^{2}}{2 m^{*}}\left(\frac{n_{x}^{2}}{L_{x}^{2}}+\frac{n_{y}^{2}}{L_{y}^{2}}+\frac{n_{z}^{2}}{L_{z}^{2}}\right),
$$

where $L_{x}, L_{y}, L_{z}$ are the dimensions of the confined structure, $m^{*}$ is the effective mass. The relation in Eq. (4) is what leads to the density of states of Fig. 1 . The valence band density of states shows a peak away from the band edge arising from the energy points where the primarily HH and LH states come close to each other. This is due to the fact that $|3 / 2, \pm 3 / 2\rangle$ (or $H H$ ) states which have no $z$ component are excited in longer structures along $z$ direction. This characteristic can be understood by examining the wave functions of the eigenstates. The peaks in Fig. 2(d) come from LH states (at $\sim 38 \mathrm{meV}$ ), higher LH and HH states with strong band mixing (at $\sim 52 \mathrm{meV}$ ) and HH states (at $\sim 61 \mathrm{meV}$ ). These results are consistent with the nonparabolic band properties in quantum wires with 

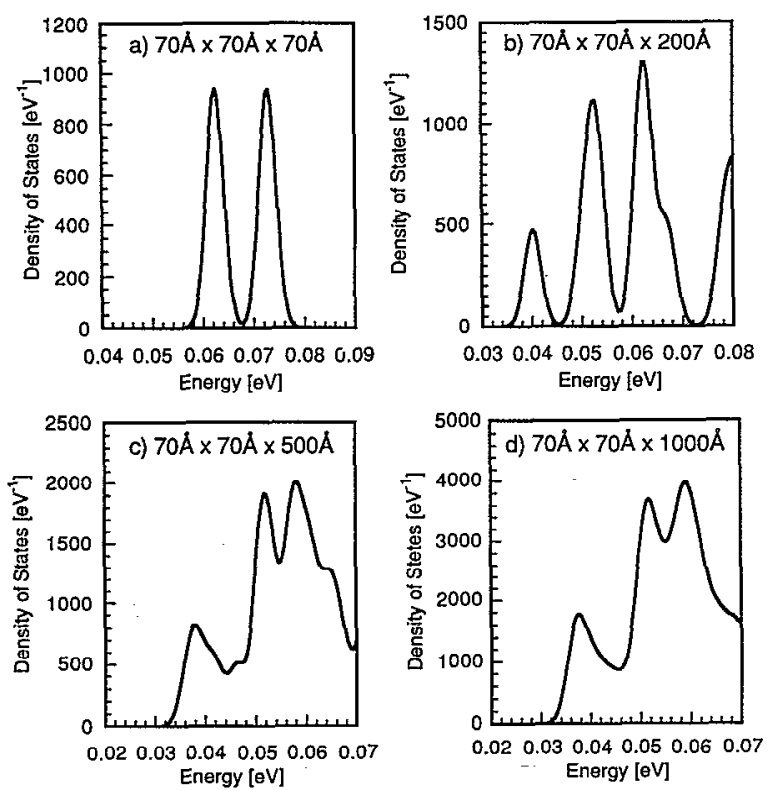

FIG. 2. The valence band density of states for the $\mathrm{GaAs} / \mathrm{Al}_{0.3} \mathrm{Ga}_{0.7} \mathrm{As}$ structures of various sizes.

infinite length. ${ }^{12,13}$ The density of states thus looks almost like a three-dimensional density of states. Note that the density of states function for the holes tends to saturate when the long axis becomes $\sim 500 \AA . \AA$. For example, the density of states for the $70 \AA \times 70 \AA \times 1000 \AA$ is simply twice that for the $70 \AA \times 70 \AA \times 500 \AA$ case.

The strong $\mathrm{HH}, \mathrm{LH}$ mixing which reflects itself in the valence band density of states is also reflected the polarization dependence of optical transitions. These results are shown in Fig. 3. In the cubic dot there is no difference between the transitions due to $x, y, z$ polarized light transition. However, as the $L_{z}$ axis increases, the low lying states have a primarily $|3 / 2, \pm 1 / 2\rangle$ (or $\mathrm{LH}$ ) character ensuring a preference for the $z$ polarization (i.e., the long axis) transitions. The excited states have a primarily a $|3 / 2, \pm 3 / 2\rangle$ (or $\mathrm{HH}$ ) character for which $z$-polarization transitions are forbidden. By the time the quantum dot long axis approaches $1000 \AA$, the optical properties look essentially like those of a quantum wire (with the line broadening included).

The case of the $70 \AA \times 100 \AA \times 200 \AA$ dot is quite interesting and must be contrasted with the dot of similar dimensions $70 \AA \times 70 \AA \times 200 \AA$. In the $70 \AA \times 70 \AA \times 200$ $\AA$ case there is no difference between the $x$ and $y$ polarization results. However, for the $70 \AA \times 100 \AA \times 200 \AA$ case there is strong anisotropy due to the loss of symmetry in the structure. It is quite clear that the polarization dependence is a very sensitive tool to study the quality of the structure fabricated.

In summary, we have solved the full three-dimensional Schrödinger equation including the $\mathrm{HH}-\mathrm{LH}$ mixing. The results are used to study the polarization dependence of the optical transitions on the symmetry of the quantum dot structure. The polarization dependence is found to be very sensitive to the symmetry of the confining structure. In
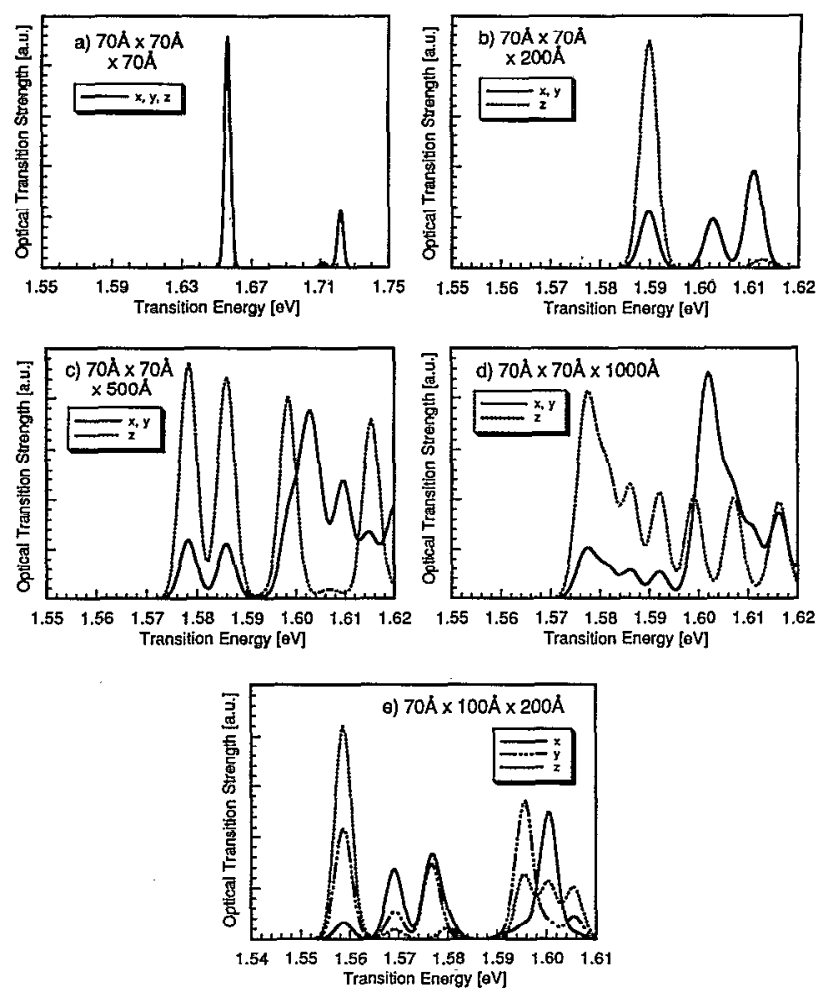

FIG. 3. Polarization dependence of the optical transitions. (a) $70 \AA \times 70$

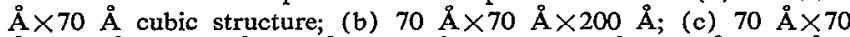
$\AA \times 500 \AA$; (d) $70 \AA \times 70 \AA \times 1000 \AA$; and (e) $70 \AA \times 100 \AA \times 200 \AA$.

addition, we examine how the optical properties of the quantum dot evolve towards those of a quantum wire by allowing one axis of the dot to increase.

This work is supported by the University-Industry Joint Project on Mesoscopic Electronics, a Grant-in-Aid for Scientific Research on Priority Area, "Electron Wave Interference Effects in Mesoscopic Structures" from the Ministry of Education, Science and Culture, and TEPCO Research Foundation. One of us (J.S.) is grateful to the NTT endowed chair program at the University of Tokyo for sponsoring this work. The work at University of Michigan was supported by the U.S. Army URI program.

${ }^{1}$ Y. Arakawa and H. Sakaki, Appl. Phys. Lett. 40, 939 (1982).

${ }^{2} \mathrm{M}$. Asada, Y. Miyamoto, and Y. Suematsu, IEEE J. Quāintum Electron. QE-22, 1915 (1986).

${ }^{3}$ I. Suemune and L. A. Coldren, IEEE J. Quantum Electron. QE-24, 1778 (1988).

${ }^{4}$ J. Singh, Appl. Phys. Lett. 59, 3142 (1991).

${ }^{5}$ J. S. Weiner, D. A. B. Miller, D. S. Chemla, T. C. Damen, C. A. Burrus, T. H. Wood, A. C. Gossard, and W. Wiegmann, Appl. Phys. Lett. 47, 1148 (1985)

${ }^{6}$ S. Hong and J. Singh, Superlattice Microstruct. 3, 645 (1987).

${ }^{7}$ M. Tanaka and H. Sakaki, Appl. Phys. Lett. 54, 1326 (1989).

${ }^{8}$ M. Kasu, H. Ando, H. Saito, and T. Fukui, Appl. Phys. Lett. 59, 301 (1991).

${ }^{9}$ J. M. Luttinger and W. Kohn, Phys. Rev. 97, 869 (1955).

${ }^{10} \mathrm{~S}$. Hong, M. Jaffe, and J. Singh, IEEE J. Quantum Electron. QE-23, 2181 (1987).

${ }^{1}$ P. Lawaetz, Phys. Rev. B 4, 3460 (1971).

${ }^{12}$ P. C. Sercel and K. J. Vahala, Phys. Rev. B 42, 3690 (1990).

${ }^{13}$ Y. Arakawa, T. Yamauchi, and J. N. Schulman, Phys. Rev. B 43, 4732 (1991). 